\title{
Caractéristiques Dimensionnelles Des Principaux Organes Du Tractus Génital Mâle De Anisopteromalus Calandrae Howard (Hymenoptera: Pteromalidae) En Fonction De L'âge
}

\author{
Kafui Yolande Ella Anani, \\ Abla Déla Mondedji, \\ Komina Amevoin,
}

Laboratoire d'Entomologie Appliquée (LEA), Département de Zoologie et de Biologie Animale, Faculté des Sciences, Université de Lomé, Togo

\section{Christophe Bressac,}

Département professionnel Agrosciences - filière Production animale de 1'Institut de Recherche sur La Biologie de l'Insecte (IRBI), Université

François Rabelais de Tours-France

\section{Isabelle Adolé Glitho,}

Laboratoire d'Entomologie Appliquée (LEA), Département de Zoologie et de Biologie Animale, Faculté des Sciences, Université de Lomé, Togo

\section{Doi: 10.19044/esj.2018.v14n12p48 URL:http://dx.doi.org/10.19044/esj.2018.v14n12p48}

\begin{abstract}
The reproductive capacity of the parasitoïd of cowpea bruchids Anisopteromalus calandrae Howard (Hymenoptera: Pteromalidae) male depends on the number of spermatozoa produced and spilled in the seminal vesicle. The objective of this study is to determine the dimensional characteristics of the different parts of the male genital tract of A. calandrae depending on age. Photographs of the parts of the male genital tract of the nymph and the virgin adults of 0-1-5-10- and 15-day old were taken by light microscopy. The dimensional characteristics of the organs of the genital tract of $A$. calandrae has shown that when the surface of the testes decreases, that of the anterior portion of the seminal vesicle increases and the ratio between the width of the light and the total width of the accessory gland increases from emergence to the fifteenth day. The dimension of the testes decreases in favor of that of the seminal vesicle and the accessory gland of $A$. calandrae male depending on age.
\end{abstract}

Keywords: Anisopteromalus calandrae, dimensional characteristics, testes, seminal vesicle, accessory glands 


\section{Résumé}

La capacité reproductrice du mâle de Anisopteromalus calandrae Howard (Hymenoptera: Pteromalidae), un parasitoïde des bruches du niébé, dépend essentiellement du nombre de spermatozoïdes produits et déversés dans la vésicule séminale. L’objectif de cette étude est de déterminer les caractéristiques dimensionnelles des différentes parties du tractus génital mâle de $A$. calandrae en fonction de l'âge. Des photos des parties du tractus génital mâle de la nymphe et des adultes vierges de $0,1,5,10$ et 15 jours du parasitoïde ont été prises en microscopie photonique. La caractérisation dimensionnelle des organes du tractus génital de $A$. calandrae a montré que lorsque la surface des testicules diminue, celle de la portion antérieure de la vésicule séminale augmente et le rapport entre la largeur de la lumière et la largeur totale de la glande accessoire augmente de l'émergence au quinzième jour. La dimension du testicule diminue au profit de celles de la vésicule séminale et de la glande accessoire de $A$. calandrae mâle en fonction de l'âge.

Mots-clés: Anisopteromalus calandrae; caractéristiques dimensionnelles; testicules; vésicule séminale; glandes accessoires

\section{Introduction}

La protection des cultures permet d'améliorer le rendement et la qualité des denrées alimentaires. Pour cela, l'agriculteur dispose d'un éventail de méthodes de lutte pour préserver les cultures et les stocks de denrées récoltées. Parmi ces techniques, la lutte biologique qui utilise les ennemis naturels à l'instar des parasitoïdes (Mondédji et al., 2002 ; Masry et al., 2018), reste l'une des méthodes efficaces d'intervention contre des ennemis de cultures (Cameron et Walker, 2002) et des stocks (Chaisaeng et al., 2010). L'efficacité de cette méthode biologique nécessite la mise en place des conditions favorables pour assurer la pérennité de l'espèce, qui dépend entre autres de la capacité du mâle à transmettre un nombre suffisant de spermatozoïdes ayant un bon pouvoir fécondant à la femelle (Do Thi Khanh, 2005 ; Do Thi Khanh et al., 2005). Cette capacité reproductrice du parasitoïde mâle peut être influencée par les facteurs environnementaux entre autres la taille de l'hôte duquel il est issu (Lacoume et al., 2006), les substances chimiques (Lacoume et al., 2009), les chocs thermiques (Lacoume et al., 2007 ; El-Sabrout et Bressac, 2012), et l'âge de l'individu (Bressac et al., 2009).

Chez l'espèce $A$. calandrae comme chez d'autres Hyménoptères, les individus femelles sont obtenus à partir de la fécondation et les mâles sont issus de la parthénogenèse arrhénothoque (Lebreton, 2009 ; Lécureuil et al., 
2012). Ainsi, les individus femelles assurent la pérennité de l'espèce. Or, l'utilisation de $A$. calandrae dans la lutte biologique, permet de contrôler des Coléoptères qui infestent des stocks de céréales et de légumineuses (Cassi, 2017 ; Chaisaeng et al., 2010). Afin de mieux cerner le pouvoir fécondant du mâle de $A$. calandrae, il s'avère nécessaire d'étudier la variation de la taille des principaux organes constituant le tractus génital mâle de $A$. calandrae dans le but d'assurer la pérennité de l'espèce par la production d'individus femelles. L'objectif de cette étude est de déterminer les caractéristiques dimensionnelles des principaux organes (testicules, vésicules séminales et glandes accessoires) du tractus génital mâle de $A$. calandrae en fonction de l'âge.

\section{Matériel et méthodes}

\section{Matériel végétal: Vigna unguiculata (L.) Walp}

Les graines de niébé Vigna unguiculata (variété «black eyes ») avec un cycle qui varie de 70 à 140 jours du semis à la maturation des graines, ont été utilisées pour l'expérimentation. La souche utilisée, a été approvisionnée en Côte d'Ivoire.

\section{Matériel animal}

\section{Le ravageur: Callosobruchus maculatus Fabricius (Coleoptera : Chrysomelidae)}

La souche utilisée a été approvisionnée au Bénin. Les individus de cette bruche qui a servi au cours de l'expérimentation, ont été les larves âgées de 18 jours (quatrième stade larvaire).

\section{Le parasitö̈de: Anisopteromalus calandrae Howard}

La souche utilisée a été approvisionnée en Côte d'Ivoire. Les individus de $A$. calandrae qui ont servi au cours de cette expérimentation, ont été des nymphes et des adultes élevées à $28^{\circ} \mathrm{C}$.

\section{Méthodologie}

Les essais ont été réalisés conjointement dans le laboratoire de l'IRBI (Institut de Recherche sur La Biologie de 1'Insecte) à l'Université François Rabelais de Tours en France et au laboratoire de biologie cellulaire et de Microscopie électronique du CHRU de Bretonneau à Tours (France).

\section{Elevage des insectes au laboratoire \\ Elevage des bruches}

Des graines saines du niébé ont été mises en présence de bruches adultes mâles et femelles pendant 48 heures dans une chambre climatique (12: $12 \mathrm{~h} \mathrm{LD}$ à $28^{\circ} \mathrm{C}$ en continu et entre 50 et $60 \% \mathrm{HR}$ ) afin de permettre la ponte des femelles sur les graines. Les graines infestées par les bruches ont été 
conservées dans la chambre climatique pendant 18 jours à $30^{\circ} \mathrm{C}$ permettant d'obtenir des larves du $4^{\text {ème }}$ stade $\left(\mathrm{L}_{4}\right)$. Ces graines ont été conservées au réfrigérateur à $4{ }^{\circ} \mathrm{C}$ pour arrêter la croissance des larves (Do Thi Khanh, 2005).

\section{Elevage de A. calandrae}

Une colonie de $A$. calandrae a été introduite dans une cage contenant des graines de niébé infestées de larves du $4{ }^{\text {ème }}$ stade de $C$. maculatus pour la ponte pendant 48 heures. Les graines ont été ensuite récupérées et conservées jusqu'à l'émergence d'adultes de parasitoïde $A$. calandrae pour le maintien de l'élevage et les différentes expérimentations.

\section{Préparation des patchs de ponte}

Chacune des femelles de $A$. calandrae âgée de moins de 24 heures a été mise en ponte dans une boîte de pétri en plexiglas de $9 \mathrm{~cm}$ de diamètre, contenant 7 graines et du coton imbibé d'eau sucrée pendant 24 heures. Chaque graine a contenu une à quatre larves du $4{ }^{\text {ème }}$ stade (âgées de 18 jours) de $C$. maculatus fixées au fond de la boîte de pétri par de la patafix. Après ce séjour de 24 heures, les graines mises en contact des femelles de $A$. calandrae ont été récupérées et introduites dans une autre boîte de pétri pendant 72 heures.

\section{Dissection des graines}

Après 72 heures, les graines ont été disséquées sous une loupe binoculaire afin de récupérer les larves du deuxième stade $\left(\mathrm{L}_{2}\right.$ ou âgées de 4 à 5 jours) du parasitoïde. Chaque larve est placée dans un puits d'une plaque de développement jusqu'à l'obtention des nymphes ou d'adultes.

\section{Dissection des individus mâles de $\boldsymbol{A}$. calandrae}

Trente (30) nymphes mâles et 30 adultes mâles de chaque âge ( 0 jour, 1 jour, 5 jours, 10 jours et 15 jours) ont été disséqués sur une lame dans une solution de Hyes $\left(0,9 \% \mathrm{NaCl} ; 0,02 \% \mathrm{KCl} ; 0,02 \% \mathrm{CaCl} 2 ; 0,01 \% \mathrm{NaHCO}_{3}: \mathrm{pH}\right.$ $8,5)$.

\section{Prise des mesures des organes génitaux}

Une fois des photos prises en microscopie photonique, certains organes tels les testicules, les vésicules séminales et les glandes accessoires ont été mesurés grâce à un logiciel «Image $J$ » version 1.4.3.67 qui exprime les données en pixels. Ces données ont été après converties en $\mu \mathrm{m}$. Ensuite, l'aire des testicules et des parties antérieures des vésicules séminales puis le rapport entre la lumière (zone de réserve de sécrétion) et la largeur totale de la glande accessoire ont été calculés. 


\section{Analyse des données}

Les résultats obtenus ont été analysés grâce au logiciel SPSS version 16.0. Les moyennes ont été comparées par l'analyse de la variance (ANOVA) et ont été discriminées à l'aide du test LSD au seuil de 5\%.

\section{Résultats}

Nos résultats ont montré que la forme du tractus génital mâle de $A$. calandrae varie avec l'âge (Figures 1). Lorsque la surface des testicules de l'espèce diminue, celle des vésicules séminales et le rapport ente la largeur de la lumière et la largeur totale des glandes accessoires augmentent en fonction de l'âge.

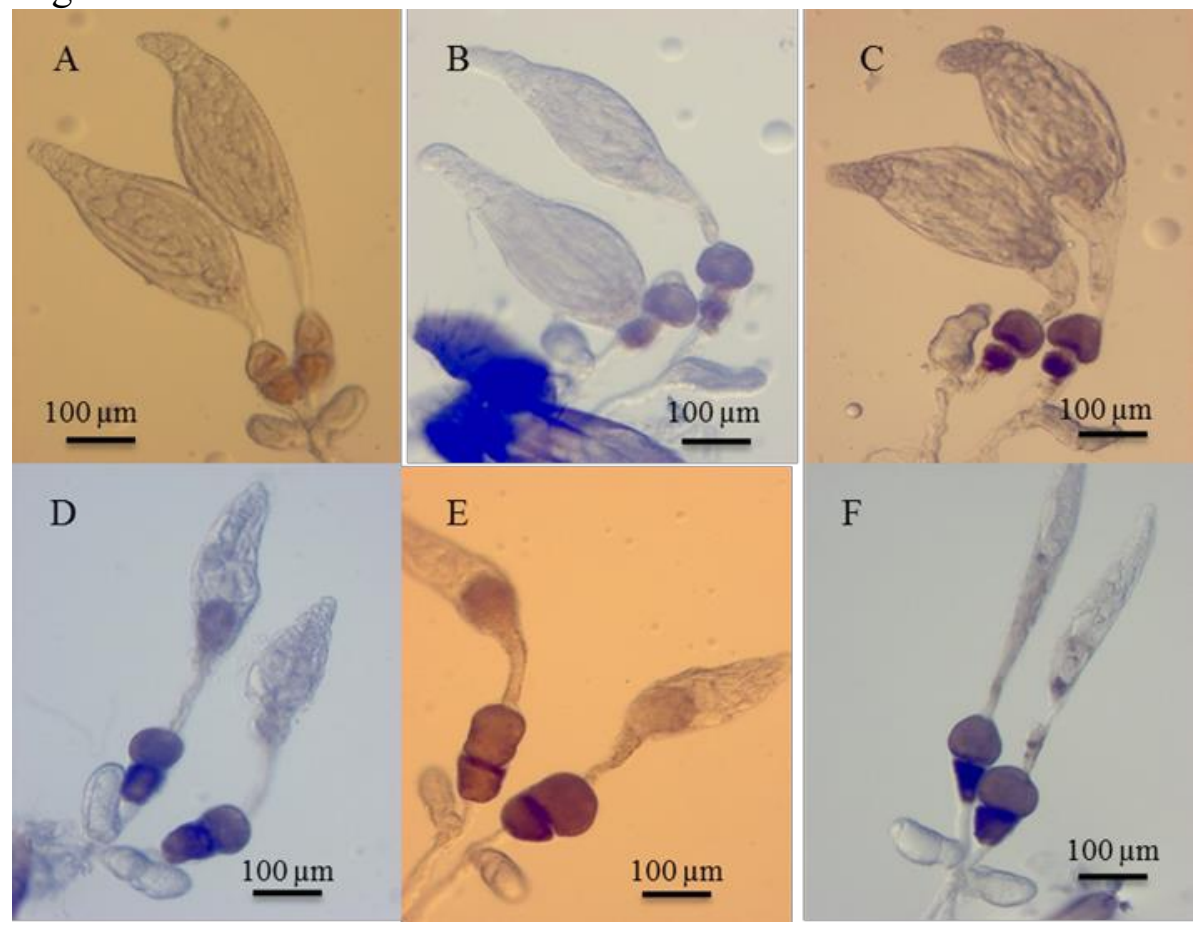

Figure 1: Tractus génital de mâle de $A$. calandrae élevé à $28^{\circ} \mathrm{C}$ et observé au microscope photonique

A-nymphe, B-adulte 0 jour, C-adulte 1 jour, D- adulte 5 jours, E- adulte 10 jours, F-adulte 15 jours (Photos prises en lumière naturelle)

La figure 2 montre une diminution de la surface des testicules au fur et à mesure que l'insecte vieillit. Cette diminution n'a pas été significative entre la nymphe et l'adulte à l'émergence. Mais la différence a été significative $\left(F_{5,24}=14,53 ; \mathrm{P}<0,0001\right)$ entre la surface des testicules de ces derniers et celle des testicules de l'adulte de 1 jour d'âge. Entre les surfaces des testicules des adultes de 5, 10 et 15 jours, les différences observées n'ont pas été significatives. 


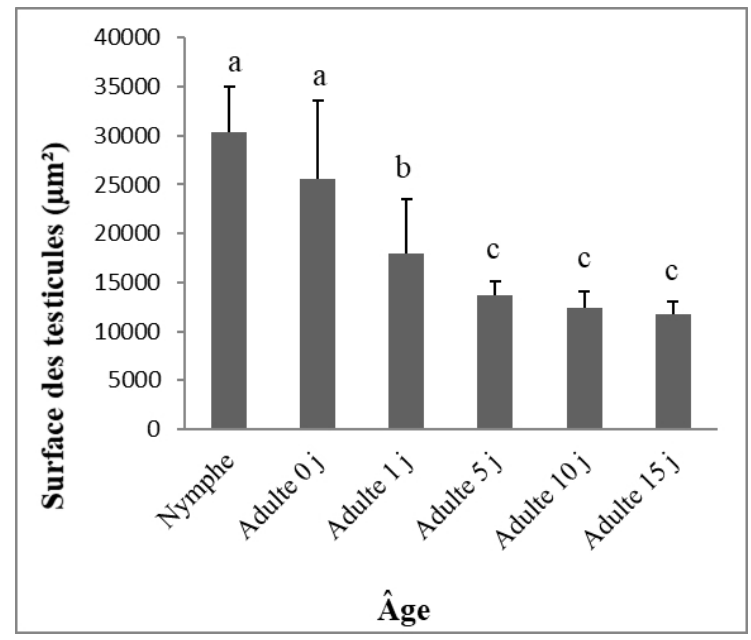

Figure 2: Evolution de la surface des testicules de $A$. calandrae en fonction du stade et de l'âge de l'adulte différentes

Les colonnes portant les mêmes lettres ne sont pas significativement

Quant à la surface des portions antérieures des vésicules séminales, elle a augmenté de manière significative $\left(\mathrm{F}_{5}, 24=5,84 ; \mathrm{P}=0,001\right)$ en fonction de l'âge de l'insecte (Figure 3). Elle a augmenté brusquement de la nymphe à l'adulte à l'émergence de l'insecte. Après émergence, la surface de cette portion de la vésicule n'a plus varié après le $5^{\text {ème }}$ jour.

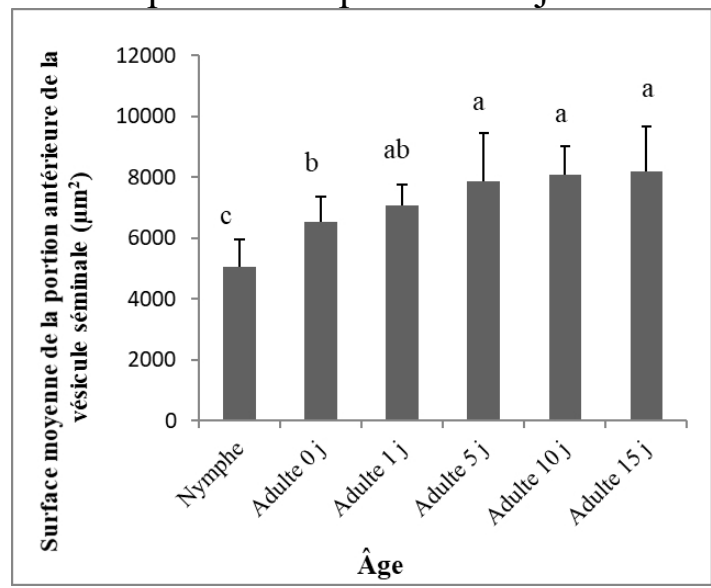

Figure 3: Evolution de la surface de la portion antérieure de la vésicule séminale de $A$. calandrae en fonction du stade et de l'âge de l'adulte

Les colonnes portant les mêmes lettres ne sont pas significativement différentes

Une corrélation négative a été observée entre la surface des testicules et la surface de la portion antérieure de la vésicule séminale (Figure 4). La diminution de la surface des testicules a été fonction de l'augmentation de la 
surface de la portion antérieure de la vésicule séminale $(\mathrm{Y}=-6,2557 \mathrm{X}+$ $\left.63276 ; \mathrm{R}^{2}=0,9577\right)$.

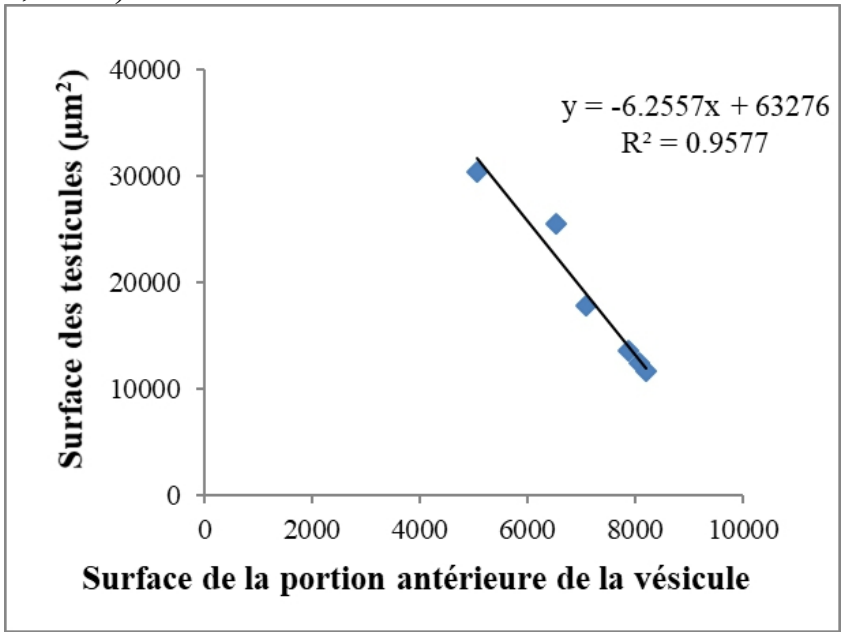

Figure 4: Corrélation entre la surface des testicules et celle de la portion antérieure de la vésicule séminale

Pour ce qui concerne la réserve de sécrétion des glandes accessoires de A. calandrae, le rapport entre la largeur de la lumière et la largeur totale de la glande accessoire a augmenté avec l'âge de manière significative $\left(\mathrm{F}_{5}, 24=\right.$ 33,55; $\mathrm{P}<0,0001)$. Juste après l'émergence, ce rapport a subitement doublé. Une évolution progressive de ce rapport a été observée dès les premières 24 heures jusqu'au $15^{\text {ème }}$ jour de la vie de l'insecte. (Figure 5).

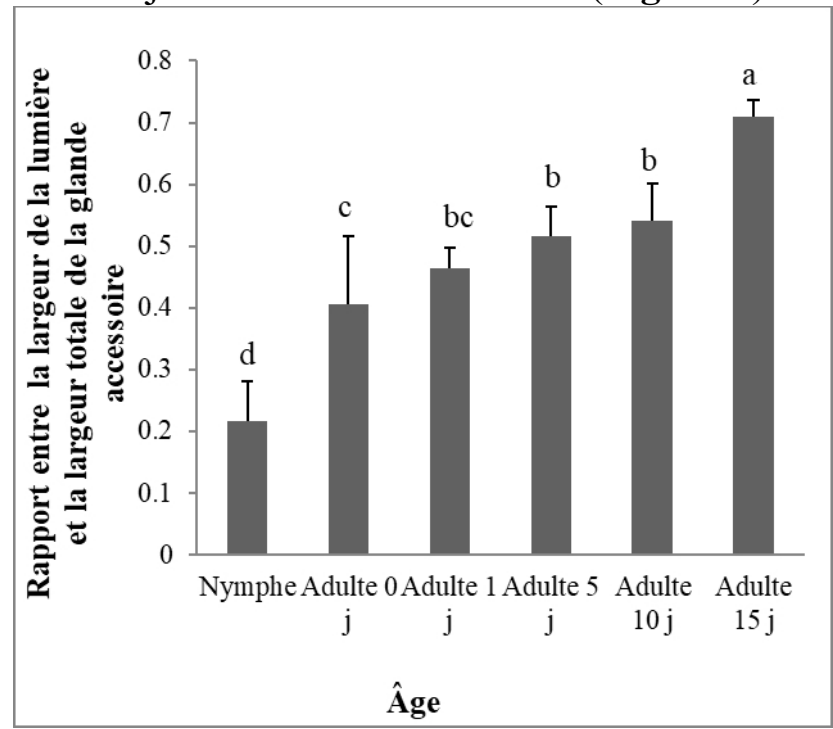

Figure 5: Rapport entre la largeur de la lumière et la largeur totale de la glande accessoire de $A$. calandrae en fonction du stade et de l'âge de l'adulte différentes

Les colonnes portant les mêmes lettres ne sont pas significativement 


\section{Discussion}

Les dimensions des différents organes du tractus génital mâle de $A$. calandrae ont varié en fonction de l'âge de l'insecte. Tous les organes n'ont pas suivi la même évolution dimensionnelle en fonction de l'âge. La surface des testicules a diminué alors que celle de la partie antérieure ou haute des vésicules séminales et le rapport entre la largeur de la lumière et la largeur totale des glandes accessoires ont augmenté avec l'âge. La diminution de la surface des testicules et l'augmentation de la surface de la partie antérieure de la vésicule séminale qui est le lieu de stockage des spermatozoïdes après l'émergence, impliquerait le début de la migration des spermatozoïdes des testicules vers la vésicule séminale comme Moreira et al., 2012 l'ont observé chez Myschocyttarus cassununga von Ihering (Hymenoptera: Vespidae). L'évolution progressive et contraire des deux organes dans les cinq (5) premiers jours après l'émergence signifierait que les spermatozoïdes auraient été transférés vers la vésicule séminale au fur et mesure qu'ils atteignent leur maturité. Quelques jours après l'émergence, la surface des testicules est devenue stable. Ceci suggèrerait la fin de la migration des spermatozoïdes vers les vésicules séminales. L'émergence impliquerait la fin de la spermatogénèse chez $A$. calandrae. Ce type de spermatogénèse n'est pas spécifique à $A$. calandrae. Le phénomène similaire est observé chez les espèces d'Hyménoptères du genre Pegoscapus, où l'émergence implique la fin de la spermatogénèse et la migration des spermatozoïdes vers la vésicule séminale (Fiorillo et al., 2008). Chez Friesella schrottkyi Friese (Hymenoptera: Apidae), les spermatozoïdes ont un développement synchronisé et murissent en même temps. Cependant, chez cette espèce de la famille des Apidae, la migration des spermatozoïdes s'effectue entre le $9^{\text {ème }}$ et le $14^{\text {ème }}$ jour après l'émergence (Brito et al., 2010). On rencontre donc deux types de spermatogénèse chez les insectes: la spermatogénèse continue où les spermatozoïdes sont produits toute la vie comme chez Drosophila melanogaster Meigen (Diptera : Drosophilidae) (Perrin-Waldemer, 1966) et la spermatogénèse synchronisée où les cellules reproductrices sont produites en même temps (Fiorillo et al., 2008; Brito et al., 2010). La spermatogénèse paraît synchronisée chez $A$. calandrae. La partie postérieure ou basse de la vésicule séminale est une zone de sécrétion (Fausto et al., 2000). Cette activité sécrétrice dépendrait soit de l'accouplement soit du risque de compétition spermatique.

Le rapport entre la largeur de la lumière et la largeur totale de la glande accessoire augmente avec l'âge (de l'émergence au $15^{\text {ème }}$ jour. Les glandes accessoires émettent des sécrétions qui se mélangent au liquide séminal et jouent un rôle important dans la fabrication des spermatophores (Odhiambo, 1969). L'étude des sécrétions qui accompagnent les spermatozoïdes lors du transfert de ceux-ci à la femelle, a été réalisée par Zizzari et al., 2014. Selon 
ces auteurs, pour augmenter le succès de la fécondation, les mâles de divers animaux (les vers de terre, les limaces de mer, les escargots terrestres, les scorpions et les salamandres) transfèrent les secrétions des glandes accessoires aux femelles séparément du sperme. Par contre chez Ceratitis capitata Wiedemann (Diptera: Tephritidae), les cellules sécrétrices des glandes

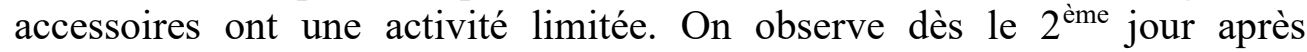
l'émergence que les cellules épithéliales de la glande accessoire deviennent moins actives. Ainsi, la production de sécrétion est réduite 10 jours après l'émergence. De plus les glandes accessoires de C capitata sont vides 30 minutes après l'accouplement (Marchini et al., 2003). Chez la mouche de fruits Bactrocera tryoni Froggatt (Diptera: Tephritidae), la sécrétion n'est pas stockée dans la lumière de la glande. Elle est transférée et expulsée vers le canal éjaculateur (Radhakrishnan et al., 2009).

Cette étude a montré que lorsque la surface des testicules diminue, celle de la partie antérieure des vésicules séminales et le rapport entre la largeur de la lumière et la largeur totale des glandes accessoires augmentent avec l'âge. L'évolution progressive et contraire des deux organes (testicules et glandes accessoires) se réalise dans les cinq (5) premiers jours après l'émergence. Cependant le rapport entre la largeur de la lumière et la largeur totale des glandes accessoires augmente de l'émergence au $15^{\text {ème }}$ jour.

\section{Conclusion}

Les caractéristiques dimensionnelles des organes du tractus génital de A. calandrae ont montré que la surface des testicules de l'espèce a diminué au profit de celle de la vésicule séminale et du rapport entre la largeur de la lumière et la largeur totale des glandes accessoires en fonction de l'âge. De l'émergence au cinquième jour, s'est réalisée une évolution progressive et contraire des testicules et des glandes accessoires. L'augmentation du rapport entre la largeur de la lumière et la largeur totale des glandes accessoires a continué quant à elle jusqu'au $15^{\text {ème }}$ jour. Ainsi, les mâles de $A$. calandrae auraient une bonne capacité à faire migrer les contenus testiculaires vers les vésicules séminales durant les cinq premiers jours de l'émergence et de produire des secrétions des glandes accessoires de l'émergence au $15^{\text {ème }}$ jour. Toute cette capacité contribuerait à augmenter le pouvoir fécondant des mâles. Les résultats de notre étude suggèrent l'étude des caractéristiques structurales de ces organes génitaux mâles pour plus d'informations quant au pouvoir fécondant de l'espèce.

\section{Remerciements}

Nos remerciements vont aux autorités de l'Université François Rabelais de Tours en France pour leur appui financier. Nos remerciements vont également à toute l'équipe d'Environnement et Hypofertilité de l'Institut 
de Recherche sur la Biologie de l'Insecte (IRBI) de la même Université pour son appui technique.

\section{References:}

1. Bressac, C., Do Thi Khanh, H. et Chevrier, C. (2009). Effects of age and repeated mating on male sperm supply and paternity in a parasitoid wasp. Netherlands Entomological Society, 130: 207-213.

2. Brito, P., Zama U., Dolder, H. et Lino-Neto J. (2010). New characteristics of the male reproductive system in the Meliponini bee, Friesella schrottkyi (Hymenoptera: Apidae): histological and physiological development during sexual maturation. Apidologie, 41: 203-215.

3. Cameron, P.J. et Walker, G.P. (2002). Field evaluation of Cotesia rubecula (Hymenoptera: Braconidae), an introduced parasitoid of Pieris rapae (Lepidoptera: Pieridae) in New Zealand. Environmental Entomology, 31 (2): 367-374.

4. Chaisaeng, P., Chongrattanameteekul, W., Visarathanonth, P. et Vajarasathiara, B. (2010). Laboratory studies on control of the maize weevil Sitophilus zeamais by the parasitoid Anisopteromalus calandrae. ScienceAsia, 36 : 6-11.

5. Do Thi Khanh, H. (2005). Anisopteromalus calandrae: un modèle pour l'étude du succès reproducteur des mâles. Thèse de Doctorat, Université de Tours, France; 154 p.

6. Do Thi Khanh, H., Bressac, C. et Chevrier, C. (2005). Male sperm donation consequences in single and double mating in Anisopteromalus calandrae. Physiological Entomology, 30: 29-35.

7. Fausto, A.M., Gambellini, G., Taddei, A.R., Maroli, M. et Mazzini, M. (2000). Ultrastructure of the seminal vesicle of Phlebotomus perniciosus Newstead (Diptera : Psychodidae), Tissue \& Cell, 32: 228237.

8. Fiorillo, B.S., Lino-Neto, J. et Bào, S.N. (2008). Stuctural and ultrastructural characterization of male reproductive tracts and spermatozoa in fig waps of the genus Pegoscapus (Hymenoptera, Chalcidoidea), Micron, 39: 1271-1280

9. Lacoume, S., Bressac, C. et Chevrier, C. (2006). Effect of host size on male fitness in the parasitoid wasp Dinarmus basalis. Journal of Insect Physiology 52 : 249-254.

10. Lacoume, S., Bressac, C. et Chevrier, C. (2007). Sperm production and mating potential of males after a cold shock on pupae of the parasitoid wasp Dinarmus basalis (Hymenoptera : Pteromalidae) . J Insect Physiol., 53 : 1008-1015.

11. Lacoume, S., Bressac, C. et Chevrier, C. (2009). Male hypofertility 
induced by Paraquat consumption in the non-target parasitoid Anisopteromalus calandrae (Hymenoptera : Pteromalidae) . Biological Control; 49 : 214-218.

12. Lebreton, S. (2009). Stratégies de ponte en situations de compétition chez une guêpe parasitoïde. Thèse de Doctorat, Université de Tours, France 144 p.

13. Lécureuil, C., Rougière, N., Nguyen, T. M., Bressac, C. et Chevrier, C. (2012). Les hyménoptères parasitoïdes : Des modèles pour l'étude de l'hypofertilité mâle. EDP Sciences, 28 (1) : 76-81.

14. Marchini, D., Del Bene, G., Cappelli, L. et Dallai, R. (2003). Ultrastructure of the male reproductive accessory glands in the medly Ceratitis capitata (Diptera: Tephitidae) and preliminary characterization of their secretions. Arthropod Structure \& Development, 31: 313-327.

15. Masry, A., Furlong, M.J., Clarke, A.R. et Cunningham, J.P. (2018). An improved culturing method for opiine fruit fly parasitoids and its application to parasitoid monitoring in the field. Insect Science, 25 (1): 99-108.

16. Mondédji, D., Amevoin, K., Nuto, Y. et Glitho, I.A. (2002). Potentiel reproducteur de Dinarmus Basalis Rond. (Hymenoptera : Pteromalidae) en présence de son hôte Callosobruchus maculatus F. (Coleoptera : Bruchidae) en zone guinéenne. Insect Science and Its Application 22 (2): 113-121.

17. Moreira, J., Brito, P., Mancini, K., Dolder, H. et Lino-Neto, J. (2012). The descriptions of new microanatomical structures of the male reproductive system and sperm of Myschocyttarus cassununga (Hymenoptera: Vespidae). Micron, 43: 292-297.

18. Odhiambo, T.R. (1969). The architecture of the accessory glands of the male dessert Locust. Tissue \& Cell, 1(1) : 155-182.

19. Perrin-Waldemer, C. (1966). Biologie et reproduction du mâle et des spermatozoïdes de Drosophila melanogaster. Annales de Biologie Animale Biochimie Biophysique. EDP Sciences, 6, (4) : 553-585.

20. Cassi, M.S. (2017). Approaches for the biological control of stored product pests. Doctoral thesis, Universitat Autonoma de Barcelona, $267 p$.

21. Radhakrishnan, P., Marchini, D. et Taylor, P. W. (2009). Ultrastructure of male reproductive accessory glands and ejaculatory duct in the Queensland fruit fly, Bactrocera tryoni (Diptera: Tephritidae. Arthropod Structure \& Development, 38: 216-226.

22. Zizzari, V., Smolders, I. et Koene, M.J. (2014). Alternative delivery of male

23. accessory gland products, Frontiers in zoology 11: 32. 